Research Paper

\title{
Knockdown of Nrf2 Inhibits the Angiogenesis of Rat Cardiac Micro-vascular Endothelial Cells under Hypoxic Conditions
}

\author{
Lihong Kuang, Jian Feng, Guoxiang $\mathrm{He}^{\bowtie}$, and Tao Jing \\ Department of Cardiology, Southwest Hospital, The Third Military Medical University, Chongqing City 400038, P. R. China.
}

$\triangle$ Corresponding author: Guoxiang He, Department of Cardiology, Southwest Hospital, The Third Military Medical University, Chongqing City 400038, P.R. China. Tel.: +86-23-68754268, Fax: +86-23-68754007, E-mail: heguoxiangcq@gmail.com; Tao Jing, Department of Cardiology, Southwest Hospital, The Third Military Medical University, Chongqing City 400038, P.R. China. Tel.: +86-23-68765172, Fax: +86-23-68754007, E-mail: xnkjt@sohu.com.

(C) Ivyspring International Publisher. This is an open-access article distributed under the terms of the Creative Commons License (http://creativecommons.org/ licenses/by-nc-nd/3.0/). Reproduction is permitted for personal, noncommercial use, provided that the article is in whole, unmodified, and properly cited.

Received: 2013.01.16; Accepted: 2013.06.28; Published: 2013.07.07

\begin{abstract}
Angiogenesis plays an important role in myocardial repair after myocardial infarction (MI). Cardiac micro-vascular endothelial cells (CMECs) are important participants in myocardial angiogenesis processes. Recent studies have revealed that Nuclear factor-erythroid 2-related factor 2 (Nrf2), a master transcription factor of endogenous anti-oxidative defense systems, exerts cardio-protection in the cardiovascular system. However, the role of Nrf2 in the process of myocardial angiogenesis and corresponding mechanisms are not fully understood. Thus, the present study investigated the role of Nrf2 in the angiogenesis of rat CMECs to hypoxia. Trans-well assay, three-dimensional Matrigel assay were used to determine cell migration and vascular tube formation. Real-time RT-PCR, ELISA and Western blot were measured mRNA and protein expression. Here, we report that the mRNA and protein expression of Nrf2 and heme oxygenase-I (HO-I) were temporarily upregulated under hypoxic condition. Furthermore, knock down of Nrf2 significantly suppressed the migration and vascular tube formation of rat CMECs to hypoxia, Nrf2 knockdown also significantly decreased $\mathrm{HO}-\mathrm{I}$ and vascular endothelial growth factor (VEGF) expression at $48 \mathrm{~h}$ after transfection under hypoxic condition. Finally, transfection of CMECs with the Nrf2 over-expressing lentiviral vector upregulated $\mathrm{HO}-\mathrm{I}$ expression with a concomitant increase in cell migration and vascular tube formation induced by hypoxia, and this effect was greatly attenuated in the presence of ZnPP (a HO-I inhibitor). Taken together, these results suggest that $\mathrm{Nrf2}$ may mediate the angiogenesis of CMECs under hypoxic condition, and $\mathrm{HO}-\mathrm{I}$ is involved in regulating the angiogenesis of CMECs through Nrf2. Therefore, Nrf2 is a potent regulator of hypoxia-condition mediated angiogenesis in CMECs, which may provide a therapeutic strategy for myocardial repair after MI.
\end{abstract}

Key words: Angiogenesis; Cardiac microvascular endothelial cell; NF-E2-related factor 2; Hypoxia.

\section{Introduction}

It is firmly established that angiogenesis, the process whereby new micro-vessels form from preexisting vessels involving the proliferation, migration, sprouting, and tube formation of endothelial cells [1], plays an essential role in many physiological and pathological processes including embryonic development, tissue repair, tumor progression and metastasis, and revascularization of the myocardium following myocardial infarction (MI) [2, 3]. Currently, angiogenesis is being investigated as a therapy for 
patients with advanced coronary artery disease (CAD). The formation of new blood vessels attributed to angiogenesis is critical for supplying the healing infarcted myocardium with oxygen and nutrients to sustain metabolism, ultimate to smaller territories of ischemic myocardium [4]. It is thought that cardiac micro-vascular endothelial cells (CMECs), a specific cell type derived from coronary micro-vessels, play a central role in the pathological processes of angiogenesis [5].

Nrf2 belongs to the Cap'n'collar (CNC) family of basic leucine zipper (bZip) transcription factor [6]. Under normal conditions, Nrf2 is sequestered in the cytoplasm by Kelch ECH associating protein 1(Keap1) and remains inactive. However, upon exposure to oxidative stress, Nrf2 is released from Keap1, tranlocates into the nucleus and stimulates antioxidant response element (ARE)-dependent phase II gene expression $[7,8]$. There is increasing evidence to suggest that the role of Nrf2 in cardio-protection, such as fighting against pathological cardiac remodeling by suppresses oxidative stress and cell death $[9,10]$, protection myocardium from ischemia / reperfusion injury by upregulating of antioxidative enzymes [11], and delay atherosclerotic lesions [12]. Moreover, the studies supporting a role for Nrf2 in angiogenesis have shown that Nrf2 may promote vascular development through protection of the retina from hyperoxia induced oxidative stress in vivo [13], and that Nrf2 knockdown inhibits colon tumor angiogenesis in vitro [14] and cervical cancer growth in vivo [15]. In addition, Pendyala et al reported that Nrf2 regulates lung micro-vascular endothelial cell migration and angiogenesis by upregulating hyperoxia-induced Nox4 expression [16]. Recent study revealed that hypoxia is able to induce Nrf2 expression in human aortic endothelial cells [17]. Though these findings suggest that Nrf2 may be closely associated with angiogenesis, but the role of Nrf2 in CMECs angiogenesis under hypoxic conditions and underlying mechanism is not clear. A better understanding of how Nrf2 can regulate the CMECs angiogenesis process may lead to novel therapeutic strategies in the treatment of myocardial repair post-MI.

The present study was designed to investigate the effect of Nrf2 on migration and tube formation in rat CMECs under hypoxic conditions, and elucidate its underlying mechanism in these processes. Our results showed that Nrf2 knockdown inhibited migration and tube formation in cultured rat CMECs under hypoxic conditions. Moreover, Nrf2 knock-down media decrease HO-1 expression. Conversely, transfection of CMECs with the Nrf2 over-expressing lentiviral vector upregulated HO-1 expression with a concomitant increase in cell migra- tion and vascular tube formation in response to hypoxia, and this effect was greatly attenuated in the presence of zincprotoporphyrin (ZnPP).

\section{Materials and Methods}

\section{Isolation and culture of CMECs}

Adult male Sprague-Dawley rats weighting between 200 and $250 \mathrm{~g}$ were obtained from the Laboratory Animal Center of southwest Hospital, and CMECs isolated as described previously $[18,19]$. Isolated CMECs were cultured in Dulbecco's modified Eagle's medium (DMEM Hyclone, USA) containing $15 \%$ fetal bovine serum (FBS Gibco, USA), 100 $\mathrm{g} / \mathrm{ml}$ streptomycin, and $100 \mathrm{U} / \mathrm{ml}$ penicillin at $37^{\circ} \mathrm{C}$ in a humidified incubator of $5 \% \mathrm{CO}_{2}$. The endothelial phenoltype was confirmed by using VIII factor (Beyotime, China) antibodies with immunocytchemistry analysis. Cells from passages 2-4 were used in all experiments. The investigation conforms to the Guide for the Care and Use of Laboratory Animals published by the US National Institutes of Health (NIH Publication No.85-23, revised 1996). The animal protocol was approved by the animal Research Committee of The Third Military Medical University.

\section{Cell transduction}

Transfection of Nrf2 shRNA(r) Lentiviral Particles (shRNA-Nrf2) (SC-156128-V, Santa Cruz, USA) and control shRNA Lentiviral Particles-A (shRNA-control) (SC-108080, Santa Cruz, USA) were performed according to the manufacturer's instructions. Control shRNAs encoded scrambled sequences that should not lead to the specific degradation of Nrf2 mRNA. CMECs were transduced with shRNA-Nrf2, shRNA-control, respectively, for $48 \mathrm{~h}$ before being used in experiments. Uninfected CMECs were used as a blank control.

The recombinant lentivirus vector encoding enhanced green fluorescent protein and Nrf2 (Len-Nrf2) and the lentivirus control vector encoding EGFP alone (Len-control) were both obtained from Neuron Biotech (Shanghai, China). CMECs were infected with lentivirus vectors at a multiplicity of infection (MOI) of 20 in six-well plates for $48 \mathrm{~h}$. The Nrf2 levels were determined by western blot analysis and real-time RT-PCR $48 \mathrm{~h}$ post-transduction.

\section{Cell migration assay}

CMECs migration was determined using a transwell chamber ( 8.0 um membrane pores, Milipore, USA) assay. Approximately $2 \times 10^{4}$ cells at $48 \mathrm{~h}$ after transfection in $200 \mu \mathrm{l}$ of serum-low medium were placed onto the upper chamber in triplicate, and 500 $\mathrm{ll}$ of DMEM with $15 \%$ FBS was placed in the lower 
chamber [20]. The plates were incubated for $12 \mathrm{~h}$ at $37^{\circ} \mathrm{C}$ in $1 \% \mathrm{O}_{2}, 5 \% \mathrm{CO}_{2}, 94 \% \mathrm{~N}_{2}$. Cells on the top surface of the membranes were removed by cotton swabs and the cells on the bottom surface were fixed in $4 \%$ paraformaldehyde and stained with Gentian violet. Migrated cells were counted under a Leica phase contrast microscope in five random high-power fields. All groups of experiments were performed in triplicate.

\section{In vitro angiogenesis assay}

In vitro endothelial cell tube formation assay in three dimensional Matrigel culture was used as a surrogate assay for angiogenic potential. Matrigel was

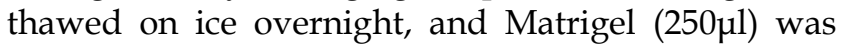
preplaced in a 24-well plate. The gel was allowed to solidify while incubating the plates for 30 minutes at $37^{\circ} \mathrm{C}$. CMECs $\left(1 \times 10^{5}\right)$ at $48 \mathrm{~h}$ after transfection with shRNA-control or shRNA-Nrf2 were transferred onto 24-well Matrigel. Endothelial tube formation was evaluated after incubation at $37^{\circ} \mathrm{C}$ in $1 \% \mathrm{O}_{2}, 5 \% \mathrm{CO}_{2}$, $94 \% \mathrm{~N}_{2}$ for $12 \mathrm{~h}$. The degree of tube formation was quantified by measurement of the length of tubes in five distinct low-power fields under an inverted phase contrast microscope using ImagePro Plus (verson 6.0). Each experiment was repeated three times, and each treatment was probed in three wells.

\section{Real-time reverse transcription-polymerase chain reaction (RT-PCR)}

Total cellular RNA was isolated using a Trizol reagent (Takara, China). One Step RT-PCR kit from Takara was performed according to the manufacture's instruction. The respective forward and reverse primers used were as follows: Nrf2, 5'-CACGGATGATGCCAGCCAG-3' (forward), and 5'-GCCCGCCCAGAAGTTCAGAGAG-3' (reverse). HO-1, 5'-CGCCCCCTTCCTGTGTCTTC-3' (forward), and 5'-CCGCCTCTACCGACCAVAGTT-3' (reverse). VEGF, 5'-GTGGAAGAAGAGGCCTGGTAATG-3' (forward), and 5'-GTCCTGCCCCATTGCTCTGT ACC-3' (reverse). Real-time RT-PCR was carried out by using the SYBR Green PCR Master Mix. PCR conditions were as follows: 40 cycles of $94^{\circ} \mathrm{C}$ for $30 \mathrm{~s} ; 57^{\circ} \mathrm{C}$ for $30 \mathrm{~s}$; and $72^{\circ} \mathrm{C}$ for $30 \mathrm{~s}$. The PCR products were analyzed by electrophoresis. The amount of target gene mRNA was normalized to the level of $\beta$-actin mRNA. Each experiment was repeated three times.

\section{Nuclear extracts and western blot analysis}

Nuclear extracts were prepared using a nuclear extraction kit according to the manufacturer's protocol. Western blot analysis was performed as described previously [21].

\section{ELISA}

VEGF protein levels were determined using a rat VEGF ELISA kit from R\&D Systems, Inc. (Minneapolis, MN). In brief, CMECs at $48 \mathrm{~h}$ after transfection with shRNA-control or shRNA-Nrf2 were growth arrested and incubation at $37^{\circ} \mathrm{C}$ in $1 \% \mathrm{O}_{2}, 5 \% \mathrm{CO}_{2}$, $94 \% \mathrm{~N}_{2}$ for $12 \mathrm{~h}$. The culture supernatants were collected, and cell numbers were counted. VEGF in the

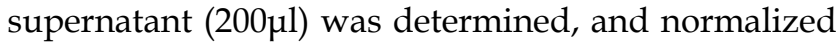
to the cell numbers.

\section{Statistical analysis}

The statistical analyses were all performed by using SPSS 17.0 software. Data are expressed as means \pm SD from three independent experiments and were analyzed by using one-way ANOVA tests. A value of $P<0.05$ was considered significant.

\section{Results}

\section{The proteins and mRNA expression of Nrf2 and HO-I were temporarily upregulated in CMECS under hypoxia conditions}

The expressions of Nrf2 and HO-1 under hypoxic conditions were studied by real-time RT-PCR and western blotting analysis. As shown in Fig.1A, after short-term exposure to hypoxia $(1 \mathrm{~h}, 2 \mathrm{~h}, 4 \mathrm{~h}, 12 \mathrm{~h}$, and $24 \mathrm{~h}$ ), we found that Nrf2 protein expression was upregulated compared to normoxia-control $(n=3, P<$ 0.05). Nrf2 protein expression was upregulated beginning at $4 \mathrm{~h}$, and increased gradually within $24 \mathrm{~h}$, the same result was shown by Nrf2 expressions at the transcriptional level ( $\mathrm{n}=3, P<0.05$, Fig.1B). Interestingly, we also found that Nrf2 protein and mRNA expression were temporarily upregulated compared to normoxia-control $(\mathrm{n}=3, P<0.05$, Fig. $1 \mathrm{C}$ and $\mathrm{D})$ in long-term exposure to hypoxia $(12 \mathrm{~h}, 24 \mathrm{~h}, 48 \mathrm{~h}$, and 72 $\mathrm{h})$, the maximum expression of Nrf2 was observed after $48 \mathrm{~h}$. Under long-term exposure to hypoxia (12h, $24 \mathrm{~h}, 48 \mathrm{~h}$, and $72 \mathrm{~h}$ ), the same result was shown by the protein and mRNA expressions of HO-1 ( $\mathrm{n}=3, \mathrm{P}<$ 0.05, Fig.1E and F). These results indicate that Nrf2 and HO-1 expression were upregulated during the early stage of cardiac adaptive hypoxia and decreased in the process of CMECs responses to long-term hypoxia.

\section{Nrf2 knockdown inhibits the migration and tube formation in CMECs to hypoxia}

The migration of CMECs plays a key role in the angiogenesis. To evaluate the effect of Nrf2 knockdown on migration of CMECs to hypoxia, trans-well assay was used to study.

First, shRNA-Nrf2 or shRNA-control was transfected into the CMECs. After $48 \mathrm{~h}$ of transfection, the 
mRNA and protein expression levels of Nrf2 were detected by real-time RT-PCR and western blot analysis. Compared with the shRNA-control, transfection with shRNA-Nrf2 significantly abated Nrf2 mRNA and protein expression at $48 \mathrm{~h}$ post-transduction $(\mathrm{n}=$ $3, P<0.05$, Fig. $2 \mathrm{~A}$ and $\mathrm{B}$ ). These results indicate that transfection with shRNA-Nrf2 was effective in rat CMECs.

Next, as shown in Fig.2C, transfection with shRNA-Nrf2 significantly decreased migration of CMECs to hypoxia $12 \mathrm{~h}$ at $48 \mathrm{~h}$ post-infection compared with the shRNA-control $(n=5, P<0.05)$. These

A

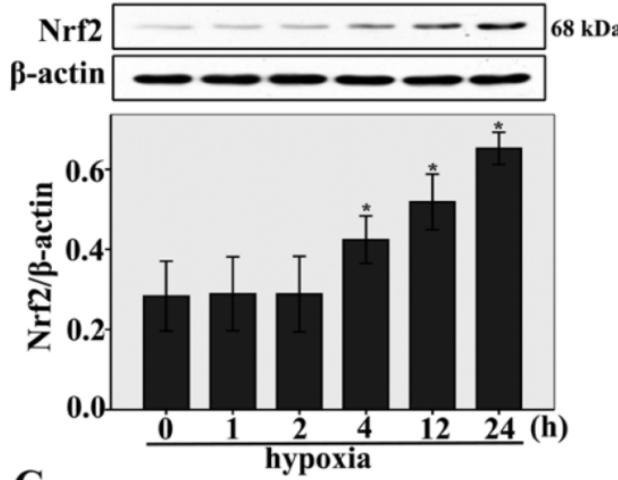

C

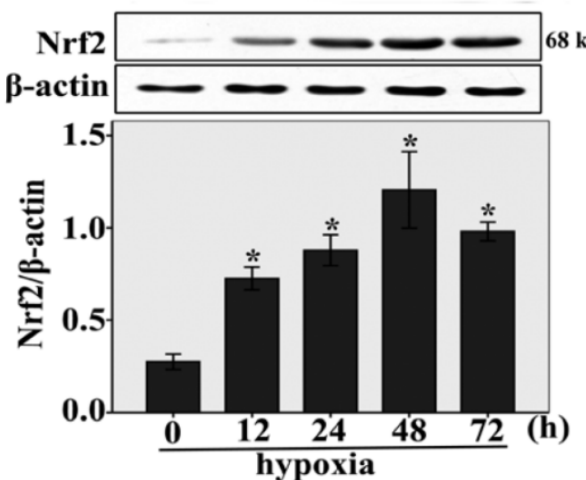

$\mathbf{E}$

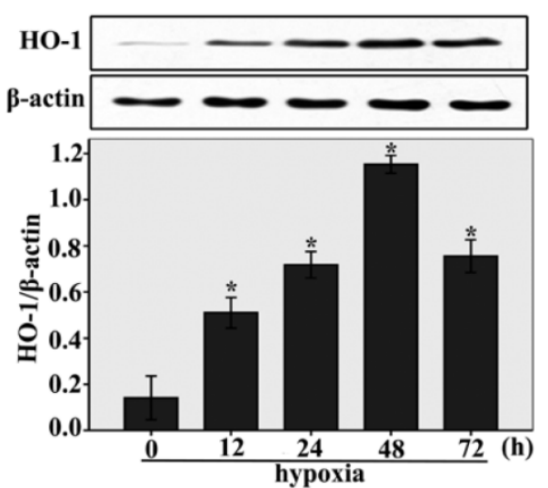

results reveal that knockdown of Nrf2 markedly inhibited the migration in CMECs to hypoxia.

Last, a three dimensional Matrigel assay was used to assess the effect of knockdown of Nrf2 on tube formation of CMECs to hypoxia. We found that transfection with shRNA-Nrf2 markedly inhibited the tube formation of CMECs to hypoxia $12 \mathrm{~h}$ compared with the shRNA-control ( $\mathrm{n}=5, P<0.05$, Fig. $2 \mathrm{D})$. These results demonstrate that Nrf2 knockdown significantly suppressed the tube formation in CMECs to hypoxia.

B

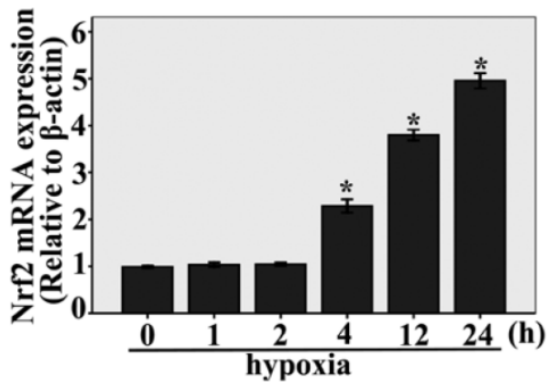

D

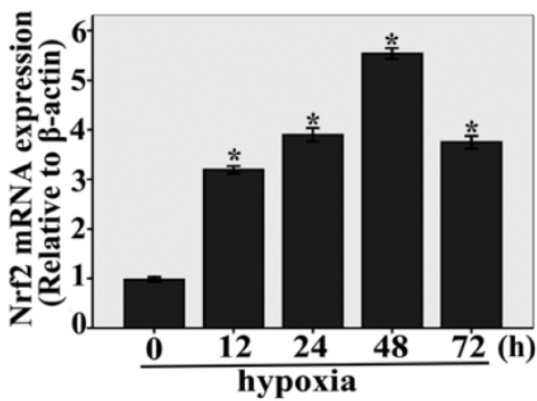

$\mathbf{F}$

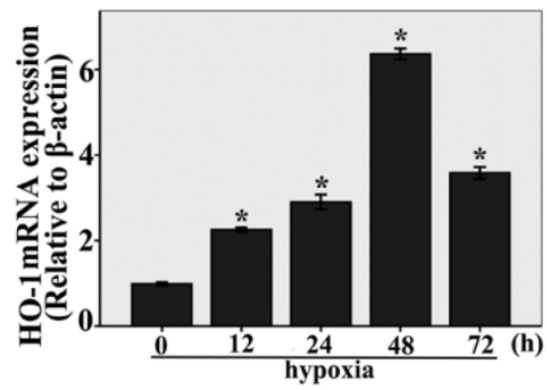

Fig I. The expressions of Nrf2 and HO-I were upregulated in CMECs incubated with hypoxia for the indicated times. A and B: Western blot and real-time RT-PCR analysis showing Nrf2 protein and mRNA levels in CMECs with short-term exposure to hypoxia (Ih, $2 \mathrm{~h}, 4 \mathrm{~h}, 12 \mathrm{~h}$, and $24 \mathrm{~h}$ ). $\beta$-actin was used as a control for equal loading. $* P<0.05$ versus normoxia. $C$ and D: CMECs were incubated with long-term hypoxia ( $12 \mathrm{~h}, 24 \mathrm{~h}, 48 \mathrm{~h}$, and $72 \mathrm{~h})$ to detect $\mathrm{Nrf2}$ protein and mRNA levels by western blot and real-time RT-PCR. $* P<0.05$ versus normoxia. E and F: Western blot and real-time RT-PCR analysis showing HO-I protein and mRNA levels in CMECs with long-term exposure to hypoxia $(12 \mathrm{~h}, 24 \mathrm{~h}, 48 \mathrm{~h}$, and $72 \mathrm{~h})$. $* \mathrm{P}<0.05$ versus normoxia. Data are expressed as the mean \pm SD of at least three independent experiments. 
A

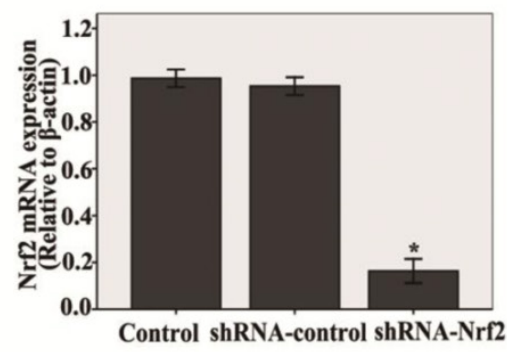

B

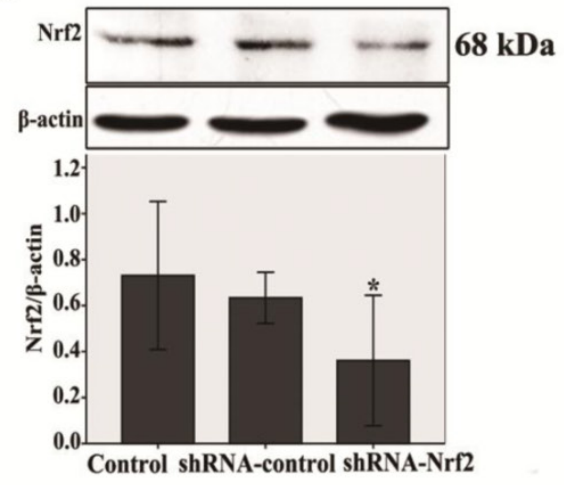

C

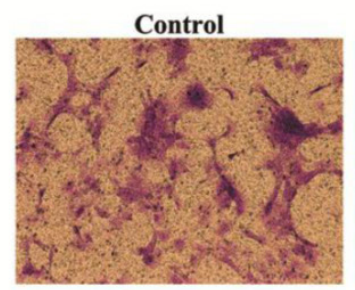

ShRNA-control
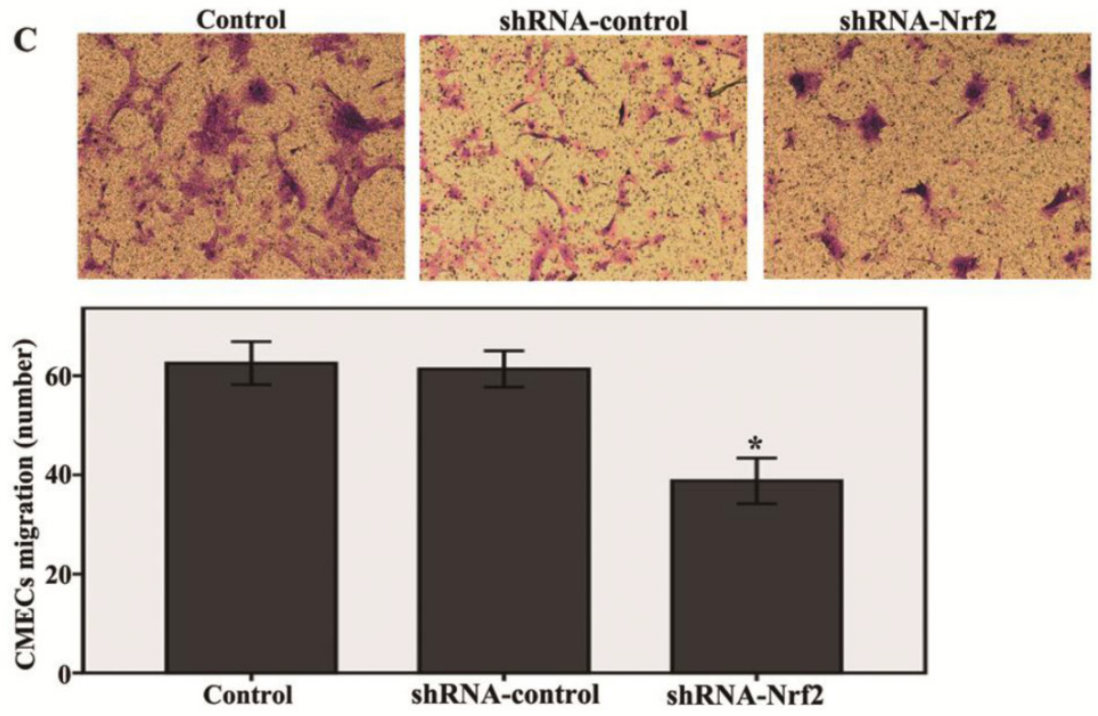

D

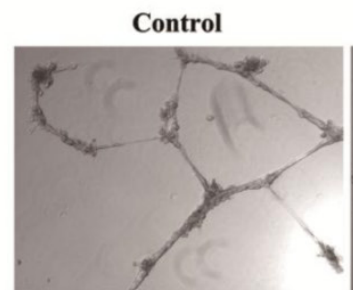

shRNA-control
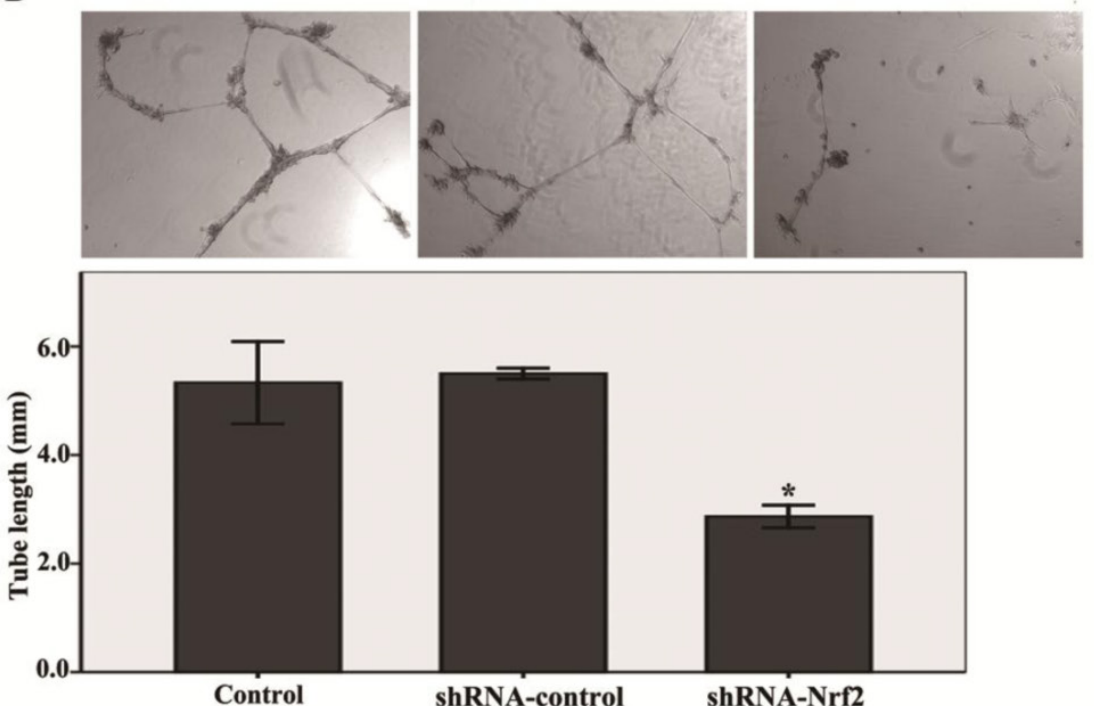

Control

\section{shRNA-Nrf2}

Fig 2. Knockdown of Nrf2 inhibits the migration and tube formation in CMECs to hypoxia $12 \mathrm{~h}$. A and B: After $48 \mathrm{~h}$ of transfection, Nrf2 levels were determined using real-time RT-PCR and western blot. Transfection of CMECs with shRNA-Nrf2 significantly decreased Nrf2 mRNA and protein expressions at $48 \mathrm{~h}$ post-transduction compared to the shRNA-controls. C: CMECs migration in expose to hypoxia for $12 \mathrm{~h}$ after transfection $48 \mathrm{~h}$ was measured using a trans-well assay (I00 $\times$ magnifications). Transfection of CMECs with shRNA-Nrf2 notably decreased the number of migrating CMECs. D: A three dimensional Matrigel assay was used to assess the effect of knockdown of Nrf2 on tube formation of CMECs to hypoxia 12h. (40 × magnifications). Transfection of CMECs with shRNA-Nrf2 significantly suppressed the tube formation of CMECs. Data are expressed as the mean \pm SD of at least three independent experiments. $* P<0.05$ versus transfection with shRNA- control CMECs. 


\section{Nrf2 knockdown impact of HO-I and VEGF on CMECs angiogenesis following to hypoxia}

To elucidate the downstream effectors of Nrf2, we examined the HO-1 and VEGF expressions. We found that knockdown of Nrf2 by shRNA significantly decreased HO-1 mRNA and protein expressions compared with the siRNA-control, as determined by real-time RT-PCR $(\mathrm{n}=3, P<0.05$, Fig.3A) and western blotting assays ( $\mathrm{n}=3, P<0.05$, Fig.3B). At the same time, as shown in Fig.3C and D, a significantly decrease in VEGF mRNA levels analysed by real-time RT-PCR ( $\mathrm{n}=3, P<0.05)$ and a slight decrease in VEGF protein levels analysed by ELISA $(\mathrm{n}=$ $3, P<0.05)$ were detected compared with the shRNA-control. Taken together, these results demonstrate that Nrf2 affects downstream target genes such as HO-1 and angiogenic growth factors VEGF in CMECs following to hypoxia.

\section{Involvement of HO-I in Nrf2 over-expressing promotes the migration and tube formation in CMECs to hypoxia}

To investigate the effect of HO- 1 on the increased migration and tube formation by Nrf2 overexpression, CMECs were treated with zinc protoporphy-

\section{A}

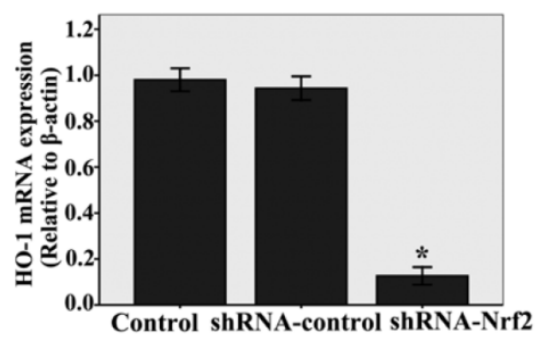

C

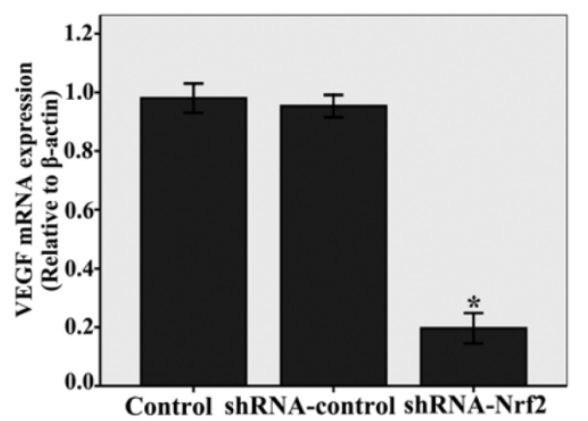

rin(ZnPP)(Sigma), an inhibitor of $\mathrm{HO}$ activity. As shown in Fig.4A and B, CMECs were transfected with either Len-control or Len- Nrf2. Nrf2 overexpression significantly increased Nrf2 mRNA and protein expression levels compared with the Len-control after 48 $\mathrm{h}$ of transfection $(\mathrm{n}=3, P<0.05)$. These results indicate that transfection with Len-Nrf2 was effective in rat CMECs. At the same time, we found that Nrf2 overexpression markedly increased HO-1 mRNA and protein expressions compared with the Len-control, as determined by real-time RT-PCR $(\mathrm{n}=3, P<0.05$, Fig.4C) and western blotting assays ( $\mathrm{n}=3, P<0.05$, Fig.4D). Furthermore, transfection with Len-Nrf2 markedly increased migration of CMECs in response to hypoxia $12 \mathrm{~h}$ compared with the Len-control $(\mathrm{n}=5$, $\mathrm{P}<0.05$, Fig.4E). The same effect was shown by tube formation of CMECs to hypoxia $12 \mathrm{~h}(\mathrm{n}=5, P<0.05$, Fig.4F). However, we found that Znpp markedly inhibited the effect of increased migration and tube formation by Nrf2 overexpression ( $\mathrm{n}=5, \mathrm{P}<0.05$, Fig.4E and F). These findings further support the notion that Nrf2 may promote migration and tube formation of CMECs to hypoxia involve transcriptional activation of HO-1.

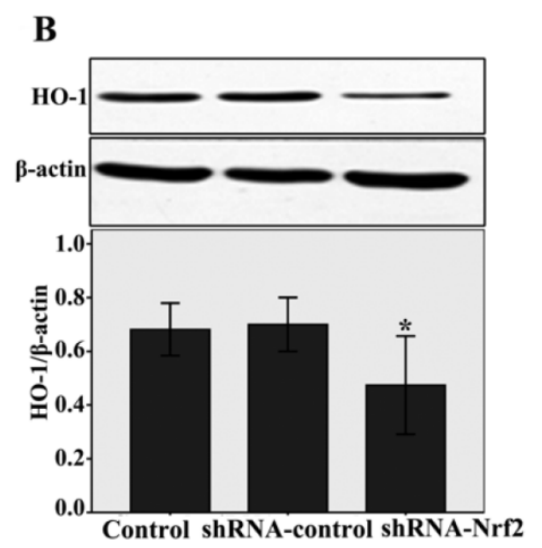

D

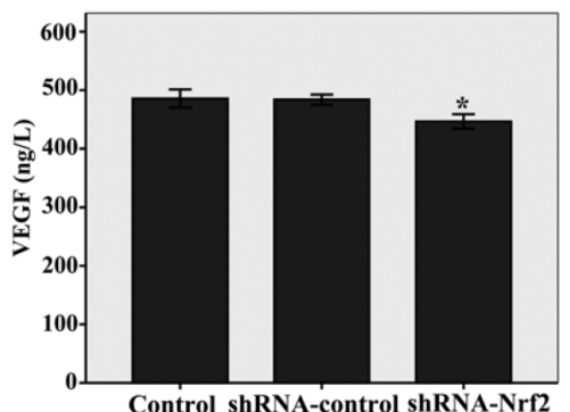

Fig 3. Nrf2 knockdown impacts of HO-I and VEGF on CMECs angiogenesis following to hypoxia. A and C: HO-I and VEGF mRNA levels were determined by real-time RT-PCR analysis. Transduction of CMECs with shRNA-Nrf2 clearly decreased HO-I and VEGF mRNA expressions under hypoxia I2 h. B and D: Western blot analysis was used to evaluate HO-I protein level, The VEGF protein level was evaluated by ELISA. Transduction of CMECs with shRNA-Nrf2 also significantly decreased HO-I and VEGF protein expressions under hypoxia $12 \mathrm{~h}$. Data represent the mean \pm SD from three different experiments. $* P<0.05$ versus transfection with shRNA- control CMECs. 
A

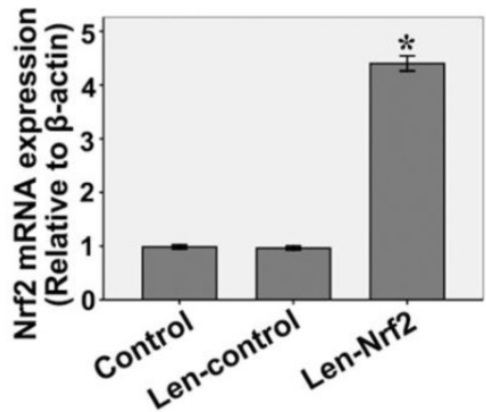

C

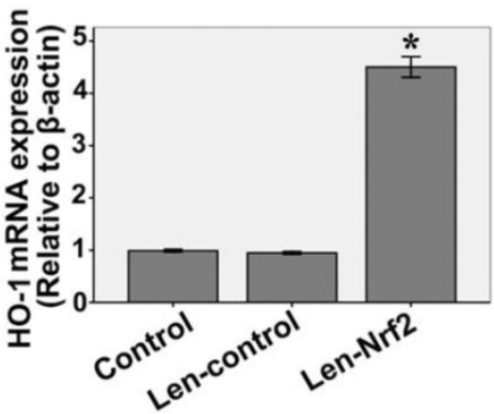

E

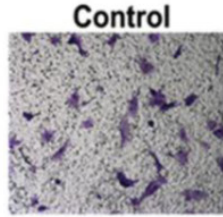

Len-Nrf2

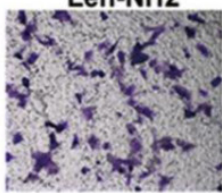

F

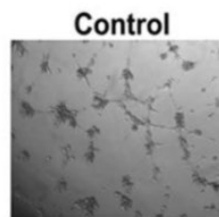

Len-Nrf2

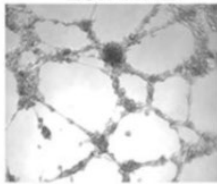

Len-control

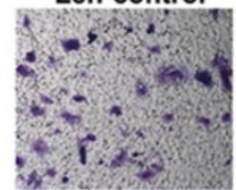

Len-Nrf2+ZnPP

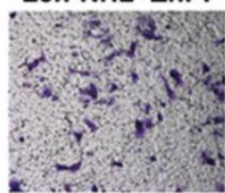

Len-control

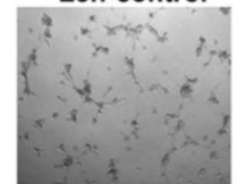

Len-Nrf2+ZnPP

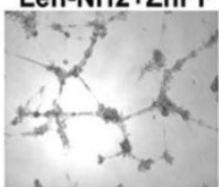

B

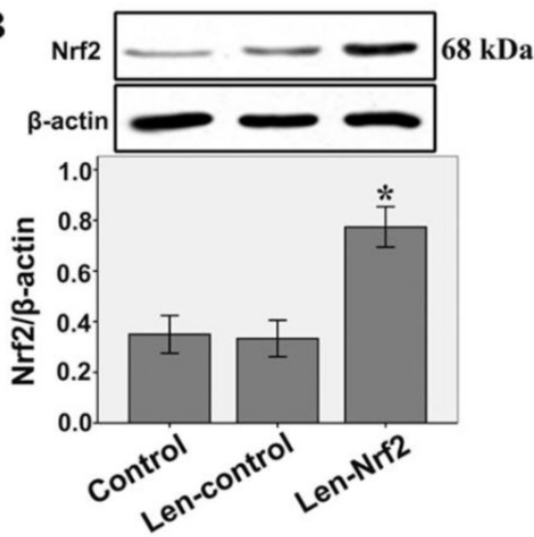

D
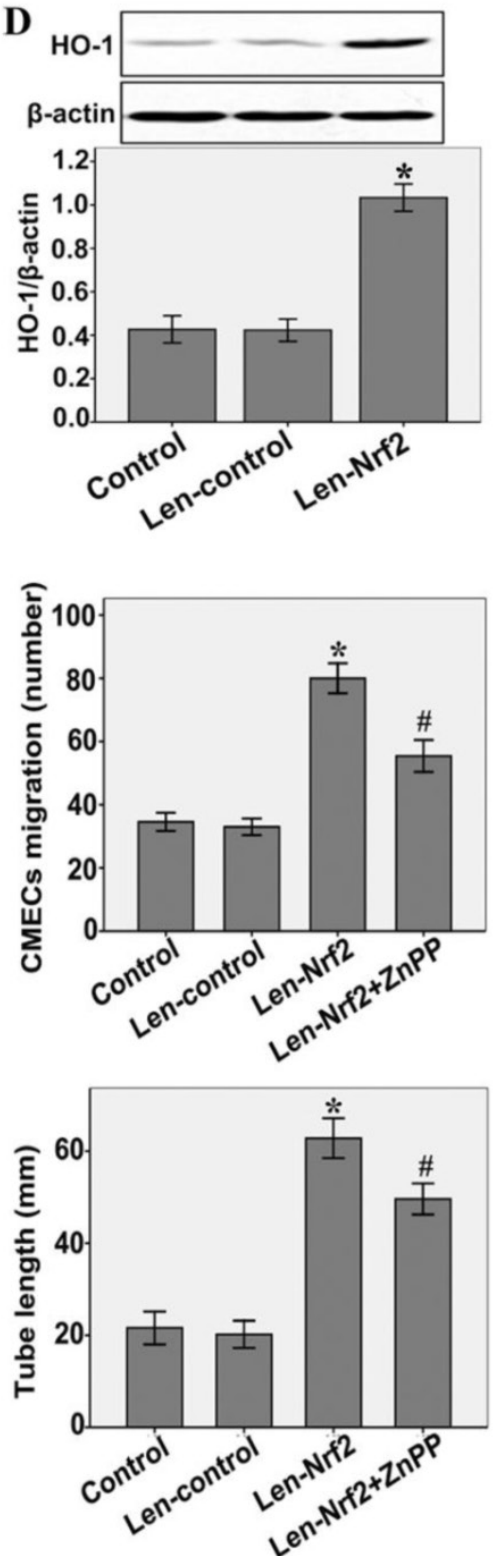

Fig 4. Nrf2 overexpression increases hypoxia-induced migration and tube formation in CMECs via modulation of heme oxygenase-I expression. A and B: Cells were transfected with Len-Nrf2 or Len-control for $48 \mathrm{~h}$, real-time RT-PCR and western blot showing Nrf2 mRNA and protein levels significantly increased in CMECs transfected with Len-Nrf2. C and D: Transduction of CMECs with Len-Nrf2 markedly increased HO-I mRNA and protein expressions compared with the Len-control, as determined by real-time RT-PCR and western blotting assays. $* P<0.05$ versus transfection with Len-control CMECs. E and F: CMECs were transfected with Len-Nrf2 in the presence or absence of ZnPP, cell migration and tube formation were assessed by trans-well assay and Matrigel assay. CMECs migrating and tube formation were significantly increased by Len-Nrf2, whereas Znpp reversed the effects of Nrf2 overexpression. Data are expressed as the mean $\pm S D$ of at least three independent experiments. $* P<0.05$ versus transfection with Len-control CMECs. $\# P<0.05$ versus transfection with Len-Nrf2 CMECs. 


\section{Discussion}

In the present study, we investigated the role of $\mathrm{Nrf} 2$ in the regulation of the processes of angiogenesis in CMECs to hypoxia. The principal new finding demonstrated that Nrf2 is a powerful regulator of the processes of angiogenesis in CMECs to hypoxia. Our results show that the Nrf2 and HO- 1 expressions were temporarily increased under hypoxic conditions. Knockdown of Nrf2 by Lentiviral delivery of shRNA significantly suppressed the migration and tube formation of CMECs to hypoxia. Nrf2 knockdown sequentially downregulate $\mathrm{HO}-1$ and proangiogenic factor VEGF expression in CMECs to hypoxia. Nrf2 over-expressing upregulated HO-1 expression with a concomitant increase in cell migration and vascular tube formation induced by hypoxia in CMECs, and this effect was greatly attenuated in the presence of ZnPP. Collectively, these studies suggest that Nrf2 may mediate angiogenesis of CMECs under hypoxic conditions through upregulating HO-1 expressions.

Recent researches revealed that short-term exposures to hypoxia upregulated Nrf2 expression in human aortic endothelial cells in vitro [17] and rat brain in vivo [22]. Interestingly, two studies reported that Nrf2 expression was transiently increased and then declined to the basal level while impairment of cardiac function proceeded [9] and in the rat middle cerebral artery occlusion (MCAO) model [23] in vivo. However, the Nrf2 expression levels in myocardial angiogenesis post-MI are not been understood. So this study evaluated the time course expressed regularity of Nrf2 in CMECs under hypoxic conditions, mimicking the myocardial angiogenesis processes post-MI. Our results showed that Nrf2 expression was upregulated at very early date $(4 \mathrm{~h})$, then gradually increased within $48 \mathrm{~h}$, and peaked at $48 \mathrm{~h}$, finally decreased at $72 \mathrm{~h}$ under $1 \% \mathrm{O}_{2}$ condition. At the same time, we found the Nrf2 expression was increased accompany by upregulating HO-1 expression. These provided the evidence that Nrf2 expression continues to rise at very early time after MI, upregulation of $\mathrm{Nrf2}$ at early stage may bring great benefit to the myocardial repair post-MI.

Angiogenesis, the formation of new blood vessels from preexisting vasculature, is essential in the myocardial repair post-MI. All the major steps of the angiogenesis process involving proliferation, migration, sprouting, and tube formation in endothelial cells. However, although endothelial cells proliferating is common hallmark of angiogenic micro-vascular sprouts, extensive sprouts can grow for periods of time, mainly by the endothelial cell migration [24]. Recent study has also reported that Nrf2 depletion enhances platelet-derived growth factor
(PDGF)-stimulated vascular smooth muscle cell migration but not cell proliferation [25]. It is well known that angiogenesis is an innate response to decreased myocardial oxygen delivery after MI. Hypoxia is a major initiator of the angiogenic switch. Moreover, CMECs, a specific cell type derived from coronary micro-vessels, are important participants in myocardial angiogenesis processes [5]. However, there are no previous studies studying the effect of Nrf2 on angiogenesis in CMECs hypoxic condition. Although Valcarcel's [26] study demonstrated that knockdown Nrf2 impaired proliferation, migration, and tube formation in human coronary arterial endothelial cells, and imply Nrf2 pathway is essential for a healthy endothelial angiogenic response, they did not reveal the effect of Nrf2 on angiogenesis in CMECs in response to hypoxia. Our results show that knockdown Nrf2 inhibited rat CMECs angiogenic activity under hypoxia $12 \mathrm{~h}$, including cell migration and tube formation, Conversely, Nrf2 overexpressing can promote the migration and tube formation in CMECs in response to hypoxia. Recent studies also reported that Nrf2 might promote migration and tube formation in lung micro-vascular endothelial cell by upregulating Nox4 expression [16] and promote endothelial cell sprout formation by upregulating ATF4 expression [27]. In animal experiments, recent study has demonstrated that genetic depletion of Nrf2 significantly inhibited retinal deep or secondary capillaries network development, which is known to form by angiogenesis in most species [13]. These studies were suggestive of an association of Nrf2 with the regulation of angiogenesis in vitro and in vivo. As is known to all, hypoxia plays an important role in promoting tumor angiogenesis. Many studies had confirmed that knockdown of Nrf2 suppresses tumor angiogenesis [14, 15]. Moreover, hypoxia may induce myocardial angiogenesis. Therefore, therapeutic angiogenesis is a new revascularization strategy in myocardial repair post-MI. The effect of Nrf2 on cell angiogenesis under hyperoxic conditions has been demonstrated in many studies. Hyperoxia may result in lung injury and retinal blood vessel obliteration. Knockdown of Nrf2 inhibited hyperoxia-induced cell migration and capillary tube formation in HLMVECs [16]. Additionally, Nrf2 may promote deep or secondary capillaries formation in Nrf2 wild type control mice exposed to hyperoxia or normoxia, which protect the retina from hyperoxia-induced oxidative stress [13]. In conclusion, our finding show that Nrf2 may mediate CMECs angiogenesis under hypoxia 12 $\mathrm{h}$ in vitro, and imply play an important role in myocardial repair post-MI at early stage.

During oxidative stress Nrf2 transactivates a battery of anti-oxidant genes and other cytoprotective 
phase II detoxifying enzymes expression, such as HO-1. Recently, Sethy's research reported that the HO-1 expression was upregulated in rat brain under short-term chronic hypobaric hypoxia in vivo [22]. However, whether chronic hypoxia stimulated HO-1 expression in CMECs is not clear. Our results also confirm that HO-1 expression was temporarily increased in culture rat CMECs under chronic hypoxia. Recent studies underscore the critical role of HO- 1 in angiogenesis. HO-1 can induce the production of vasculoprotective cytokines and media their proangiogenic action [28]. Haeme oxygenase promotes neovascularization after critical hindlimb ischaemia in mice in vivo [29]. Knockdown of HO-1 expression decreased arsenic induced VEGF expression, cell migration, and tube formation in human micro-vascular endothelial cells [30]. Hypoxia has been established as a major inducer of VEGF gene transcription. VEGF is one of the most effective angiogenic growth factors and has been show to the master regulator of angiogenesis [31]. A recent study demonstrated that Nrf2 blockade in colon cancer cells suppressed angiogenesis and tumor growth by inhibiting the activation of HIF-1a-VEGF signaling [14]. Another research also revealed oxidized phospholipids (OxPLs) may induce angiogenesis and upregulate VEGF mRNA in endothelial cell and that these effects were inhibited by knockdown of Nrf2 [27]. So we hypothesis the effect of Nrf2 on the angiogenesis in rat CMECs to hypoxia involved upregulating HO-1 and VEGF. Data from the present study show that knockdown Nrf2 may sequentially downregulate $\mathrm{HO}-1$ and pro-angiogenic factor VEGF expression in rat CMECs under hypoxia $12 \mathrm{~h}$. Furthermore, our results show that Nrf2 over-expressing induced upregulation of HO-1 expression in CMECs. At the same time, the effect of Nrf2 over-expressing promotes angiogenesis in CMECs to hypoxia may descrease by administrating Znpp. Our results suggest that under hypoxic conditions, transcriptional activation of HO-1 is mediated, at least in part, by Nrf2. Furthermore, the Nrf2/HO-1 pathway may involve in the angiogenesis in CMECs under short-term hypoxia.

In summary, the present study identifies that $\mathrm{Nrf} 2$ as a mediator of angiogenesis in CMECs under short-term hypoxic conditions; accompany the change of HO-1 and VEGF expression. These results indicate that Nrf2 may be a novel therapeutic strategy of angiogenesis in myocardial repair post-MI. Upregulation of Nrf2 expression at early stage may bring great benefit to the myocardial repair post-MI. The area of future investigation in our laboratory could further elucidate the effect of Nrf2 on myocardial angiogenesis at long-time stage in vitro and in vivo.

\section{Acknowledgments}

This study was funded by a grant from the National Natural Science Foundation of China (81170142).

\section{Competing Interests}

The authors have declared that no competing interest exists.

\section{References}

1. Bussolino F, Mantovani A, Persico G, et al. Molecular mechanisms of blood vessel formation. Trends Biochem Sci.1997; 22: 251-6.

2. Carmeliet P. Angiogenesis in health and disease. Nat Med.2003; 9:653-60.

3. Isner JM, Losordo DW. Therapeutic angiogenesis for heart failure. Nat Med.1999; 5: 491-2.

4. Elsman P, van't Hof AW, de Boer MJ, et al. Role of collateral circulation in the acute phase of ST-segment-elevation myocardial infarction treated with primary coronary intervention. Eur Heart J.2004; 25: 854-8.

5. Balligand JL, Kelly RA, Smith TW, et al. Cardiac endothelium and tissue growth. Prog Cardiovasc Dis. 1997; 39: 351

6. Li J, Ichikawa T, Janicki JS, et al. Targeting the Nrf2 pathway against cardiovascular disease. Expert Opin Ther Targets.2009; 13: 785-94.

7. Kensler TW, Wakabayashi N, Biswal S, et al. Cell survival responses to environmental stresses via the Keap1-Nrf2-ARE pathway. Annu Rev Pharmacol Toxicol.2007; 47: 89-116.

8. Nguyen T, Nioi P, Pickett CB, et al. The NRF-2-antioxidant response element signaling pathway and its activation by oxidative stress. J Biol Chem.2009; 284: 13291-5.

9. Li J, Ichikawa T, Villacorta L, et al. Nrf2 protects against maladaptive cardiac responses to hemodynamic stress. Arterioscler Thromb Vasc Biol. 2009; 29:1843-50.

10. Li J, Ichikawa T, Jin $Y$, et al. An essential role of Nrf2 in American ginseng-mediated anti-oxidative actions in cardiomyocytes. J Ethn- opharmacol.2010; 130: 222-30.

11. Dreger H, Westphal K, Weller A, et al. Nrf2-dependent upregulation of antioxidative enzymes: a novel pathway for proteasome inhibitor- mediated cardioprotection. Cardiovasc Res.2009; 83: 354-61.

12. Dai G, Vaughn S, Zhang $Y$, et al. Biomechanical forces in atherosclerosis-resistant vascular regions regulate endothelial redox balance via phosphoinositol 3-kinase/Akt-dependent activation of Nrf2. Circ Res. 2007; 101: 723-33.

13. Uno K, Prow TW, Bhutto IA, et al. Role of Nrf2 in retinal vascular development and the vaso-obliterative phase of oxygen-induced retinopathy. Exp Eye Res.2010; 90: 493-500.

14. Kim TH, Hur EG, Kang SJ, et al. NRF2 blockade suppresses colon tumor angiogenesis by inhibiting hypoxia-induced activation of HIF-1a. Cancer Res.2011; 71: 2260-75.

15. Ma X, Zhang J, Liu S, et al. Nrf2 knockdown by shRNA inhibits tumor growth and increases efficacy of chemotherapy in cervical cancer. Cancer Chemother Pharmacol.2012; 69: 485-94.

16. Pendyala S, Moitra J, Kalari S, et al. Nrf2 regulates hyperoxia- induced Nox4 expression in human lung endothelium: Identification of functional antioxidant response elements on the Nox4 promoter. Free Radic Biol Med.2011; 50: 1749-59.

17. Polotsky VY, Savransky V, Bevans-Fonti S, et al. Intermittent and sustained hypoxia induce a similar gene expression profile in human aortic endothelial cells. Physiol Genomics.2010; 41: 306-14.

18. Chen SF, Fei X, Li SH, et al. A new simple method for isolation of micro-vascular endothelial cells avoiding both chemical and mechanical injuries. Microvasc Res.1995; 50: 119-28.

19. Wang L, Chen Q, Li G, et al. Ghrelin stimulates angiogenesis via GHSR1a-dependent MEK/ERK and PI3K/Akt signal pathways in rat cardiac microvascular endothelial cells. Peptides.2012; 33: 92-100.

20. Kuang CY, Yu Y, Guo RW, et al. Silencing stromal interaction molecule 1 by RNA interference inhibits the proliferation and migration of endothelial progenitor cells. Biochem Biophys Res Commun.2010; 398: 315-20.

21. Feng J, Zhang P, Chen $X$, et al. PI3K and ERK/Nrf2 pathways are involved in oleanolic acid-induced heme oxygenase-1 expression in rat vascular smooth muscle cells. J Cell Biochem.2011; 112: 1524-31.

22. Sethy NK, Singh M, Kumar R, et al. Upregulation of transcription factor NRF2-mediated oxidative stress response pathway in rat brain under short-term chronic hypobaric hypoxia. Funct Integr Genomics. 2011;11:119-37. 
23. Yang $\mathrm{C}$, Zhang $\mathrm{X}$, Fan $\mathrm{H}$, et al. Curcumin upregulates transcription factor Nrf2, HO-1 expression and protects rat brains against focal ischemia. Brain Res. 2009; 1282:133-41.

24. Sholley MM, Ferguson GP, Seibel HR, et al. Mechanisms of neovascularization. Vascular sprouting can occur without proliferation of endothelial cells. Lab Invest, 1984; 51: 624-634.

25. Ashino T, Yamamoto M, Yoshida T, et al. Redox-sensitive transcription factor Nrf2 regulates vascular smooth muscle cell migration and neointimal hyperplasia. Arterioscler Thromb Vasc Biol, 2013; 33:760-768.

26. Valcarcel-Ares MN, Gautam T, Warrington JP, et al. Disruption of Nrf2 signaling impairs angiogenic capacity of endothelial cells: implications for microvascular aging. J Gerontol A Biol Sci Med Sci. 2012;67:821-29.

27. Afonyushkin T, Oskolkova OV, Philippova M, et al. Oxidized phospholipids regulate expression of ATF4 and VEGF in endothelial cells via NRF2-dependent mechanism novel point of convergence between electrophilic and unfolded protein stress pathways. Arterioscler Thromb Vasc Biol.2010; 30: 1007-13.

28. Nishida M, Carley WW, Gerritsen ME, et al. Isolation and characterization of human and rat cardiac microvascular endothelial cells. Am J Physiol.1993; 264: H639-52.

29. Tongers J, Knapp JW, Korf M, et al. Haeme oxygenase promotes progenitor cell mobilization, neovascularization, and functional recovery after critical hindlimb ischaemia in mice. Cardiovasc Res.2008; 78: 294-300.

30. Meng D, Wang $X$, Chang $Q$, et al. Arsenic promotes angiogenesis in vitro via a heme oxygenase-1 dependent mechanism. Toxicol Appl Pharmacol.2010; 244: 291-9.

31. Ferrara, N. Vascular endothelial growth factor: basic science and clinical progress. Endocr Rev.2004; 25: 581-611. 